\title{
Mobile Serious Game Design Using User Experience: Modeling of Software Product Line Variability
}

\author{
https://doi.org/10.3991/ijet.v14i23.10899 \\ Chaimae Meftah ${ }^{\square}$, Asmaâ Retbi, Samir Bennani, Mohammed Khalidi Idrissi \\ Mohammed V University, Rabat, Morocco \\ chaimae.meftah@gmail.com
}

\begin{abstract}
In recent years, gamification has emerged as a new approach to increasing learner engagement. It covers a very wide range of games with very different purposes and with many fields of application. However, most of the gamification solutions proposed do not adopt the same modeling approach and little attention has been paid to mobile serious games (MSG) belonging to different pedagogical contexts. In order to overcome these difficulties, we have developed in this paper a generic model based on the Software Product Line (SPL) approach to manage the common and variable points of the MSG product set. We also focused on the User eXperience (UX) concept to study the aspects that most affect the player's experience in the context of MSGs. These aspects have been modeled in the form of features in the SPL Feature Model. MSG designers can use the model proposed during the development process, both to manage variability and to create an effective and fun learning environment.
\end{abstract}

Keywords-feature model, gamification, MSG, SPL, UX, variability.

\section{Introduction}

A serious game is an activity that combines serious intention, pedagogical, informative, communicative, marketing, ideological or training, with fun springs $[10,11]$. Indeed, a serious game can in theory be used in all areas that deviate from entertainment alone. In the literature, it is possible to distinguish five major categories of serious games according to the purpose, namely advertising games, Ludoeducational games, edumarket games, engaged games and training and simulation games. The serious gambling market is important, and the various disciplines affected are constantly increasing $[10,12]$. Thus, there are multiple serious games according to sectors, such as health, ecology, economy, job market, etc. On the other hand, a large number of research projects have set up a database to classify serious games according to three axes of thought, namely the intention, the market and the public [10].

The product line approach is a new paradigm in the context of software engineering, it brings together the development activities of a set of software belonging to a particular domain $[1,2]$. The main objective of SPL is to increase productivity and reduce production time. In fact, software construction in a particular application area 
by no longer developing each software separately, but rather by designing it from reusable elements called assets.

However, the difficulty with this approach lies in the design of an architecture that allows the definition of several products. Members of a product line are characterized by their similarities, but also by their differences, also known as points of variation [3]. In the software engineering world, differences may arise depending on technical factors (use of a particular type of hardware), commercial (creation of a limited version), cultural factors (software for several countries), etc.

UX is a concept that refers to the quality of the user's experience in any interaction situation in digital environments. It describes the overall experience experienced by the user when using an interface, a digital device or any device or service [13]. Moreover, UX marks a disciplinary, methodological and conceptual evolution in the way of designing the user relationship to the products and considering their ergonomic quality. This evolution takes into account the non-instrumental characteristics of products such as appearance, aesthetics, pleasure, emotion, etc. [13, 14]. The aim is to consider all the characteristics and needs of users when developing products, and to involve them actively in the design process. This is user-centric design.

In this paper, we propose a generic approach based on SPL and UX to collect and analyze gamification data. Using the SPL approach, it is possible to easily distinguish common and variable points while using a single complete modeling infrastructure to the entire MSG product set. On the other hand, by applying UX to the context of serious games, it is possible to determine the aspects that players find most influential in their experiences of serious games by qualitatively analyzing the data.

The rest of the paper is organized as follows. Section 2 shows the basic concepts of SPL and UX. Section 3 presents the framework for our approach. Section 4 discusses MSG modeling. Finally, Section 5 provides a conclusion and some perspectives.

\section{Fundamental Concepts}

\subsection{SPL engineering}

The development of a software product line is engineering. Therefore, SPL engineering distinguishes two levels [4,9]:

- Domain engineering: it consists of developing and building the assets that will be reused for the construction of products (develop for reuse);

- Application engineering: it consists of using the assets for the construction of a particular product or application after crystallization of the points of variation (develop through reuse). 


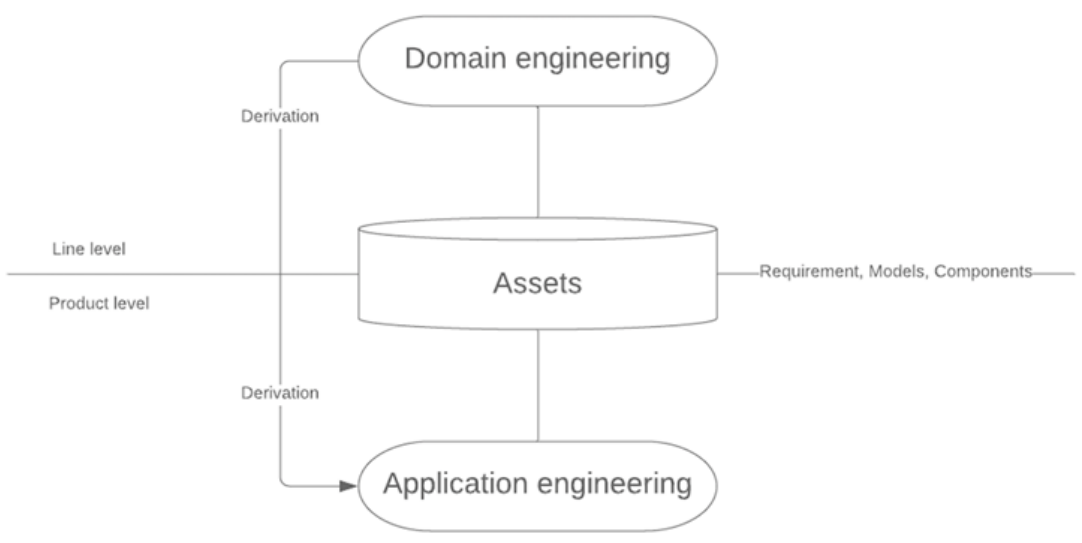

Fig. 1. Product line engineering

Useful techniques and approaches for managing variability include:

- Compilation techniques: they allow the derivation of a product during the compilation phase. Conditional compilation and library loading are examples of these techniques. They are useful if the products differ in the parts of code to include or exclude and in the libraries they use;

- Techniques related to programming languages: abstraction through the notion of polymorphism, overloading and dynamic linking. Variation points can be defined as abstract in the product line and redefined by each product in a specific way;

- Design patterns: the pattern design of Abstract Factory is used for the reification of variants. The Abstract Factory allows to define an interface for the creation of concrete products.

The techniques presented above are generally related to programming languages, and relegate the derivation activity of a product to a programming activity. But it is also important to show and specify variability on a more abstract level, especially at the model level.

The analysis phase of the domain engineering field aims to identify common and variable characteristics for the family (features) [5]. Feature Models are a technique for modeling SPLs, they were introduced for the first time in the Feature-Oriented Domain Analysis (FODA) method. In a simplified way, each product belonging to a line of software products determines a particular context, it consists of a set of software elements represented by features. The purpose of a feature is to define a set of requirements that are highly related and directly reusable by different end products. Features are successively broken down into sub-features until terminal features are obtained. Ideally, each terminal feature is associated with a reusable software element implementing the requirements determined by the corresponding feature. A Feature Model is in the form of a tree composed of nodes (features) connected by edges (relationship between two features). Each edge can have four possible values, namely Mandatory, Optional, Alternative and Or [5,8]. 


\subsection{UX and usability}

The UX represents all aspects related to the way people use an interactive product: the feeling of the product in their hands, the understanding of how it works, the feeling during use, the achievement of their goals but also its suitability with the overall context in which they use it. It is the user's feeling that distinguishes UX from usability, opening a much wider field than the proper use of an interface and linking disciplines that interpenetrate each other (user interface design, information architecture, interaction design, service design, etc.). UX is therefore not strictly pragmatic as is usability. It involves the emotional impact felt during the interaction, and even includes the anticipation of use. UX refers to the responses and perceptions of a person that result from the use or anticipation of the use of a product, service or system [13, $14,15,16]$. Figure 2 illustrates the different facets of UX.

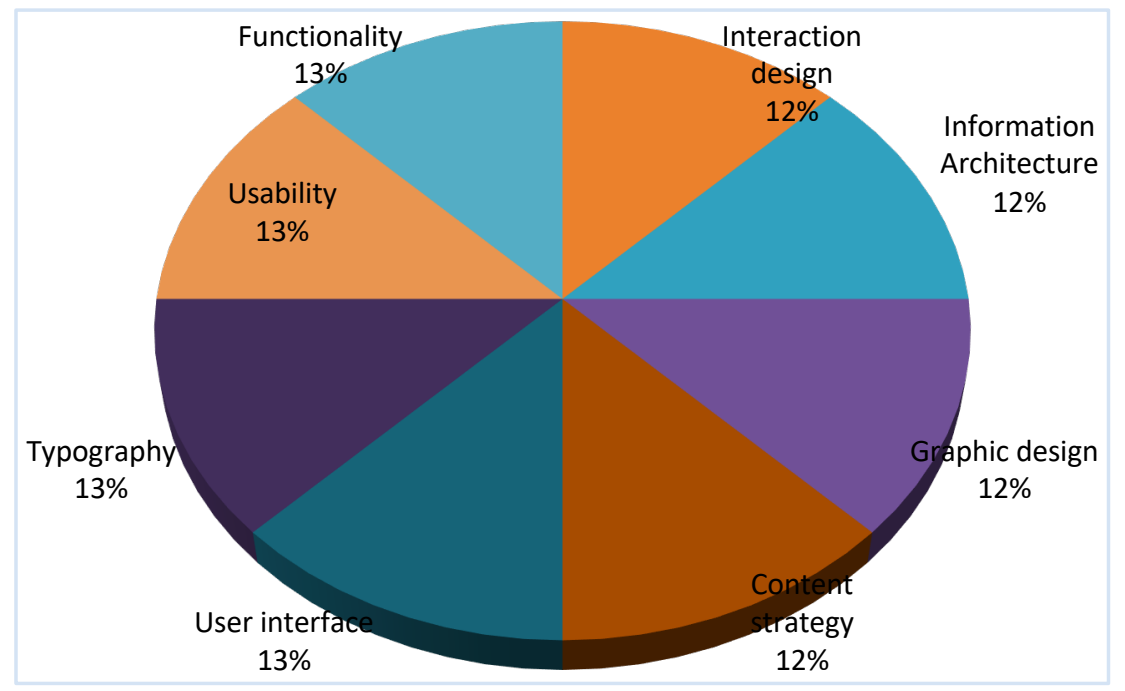

Fig. 2. Different facets of UX

The User Interface (UI) is an important element for a high-performance product. The design of a good user interface is mainly related to their graphic appearance. The user experience goes beyond; the user interface is only a component [18].

Currently, it is difficult to agree on criteria and methods for analyzing the user experience $[17,18]$. However, UX considers the following components of the different business lines:

- Emotional (image and communication professionals)

- Technical (interface performance and speed)

- Statistics (analysts and marketing experts)

- Ergonomics (for professionals in traditional ergonomics or interaction design) 
Most of the criteria are based on different data collection sources. The necessary data collection techniques can mix:

- User search (identifying expectations and cognitive barriers);

- Socio-cultural studies (expectations and needs in the face of the competitive environment and changing uses);

- Consumer perception (reception/understanding of messages, and impact of awareness);

- Performance (dynamic statistical data collected on the device, conversion);

- The exhibition (calendar, media coverage or push campaign of offers).

\section{Proposed Approach}

Feature Model is characterized and identified by a specific feature called the root (root). This root determines the entry point of the diagram which is the unique knot not possessing parent. In our example, the root is the Mobile feature Serious Game (MSG) situated at the top of the tree and which represents the line of products $[7,8]$.

The MSG is relatively similar for all the organizations, because its basic features remain identical. However, a specific version of the game must be often created for each of her [6]. Thus the software of departure is configured according to the clean requirements of the organization. All the products of the software part have common characteristics such as contexts, rules, objectives, player and tasks, which are modeled as compulsory functions. Given that a single kind (for example, education, health, politics, etc.) can be used for a product, the characteristics are modeled as alternative features. Indeed, rules can be organized by means of scores, by means of timers or by means of instructions of game to indicate to the players what must be carried out during the game and to explain in a sufficient way the objectives and the manners to play and to learn. By implementing techniques of training, motivation and the progress, the MSG will contain a realistic environment in which the users can form their own social behavior. Besides, every game contains a set of levels (novice, advanced and expert), these levels contain in their turn realizations and every realization can require several tasks, a task can be in the form of the riddles, questions or simulations. Besides, every player is characterized by his psyche, his habits and his character which influence obviously his attachment in a serious game. In our case, we use Feature Model to model in a more compact way this line of products as well as his variability. The figure 3 illustrates the software part of the MSG Feature Model under Feature IDE: 


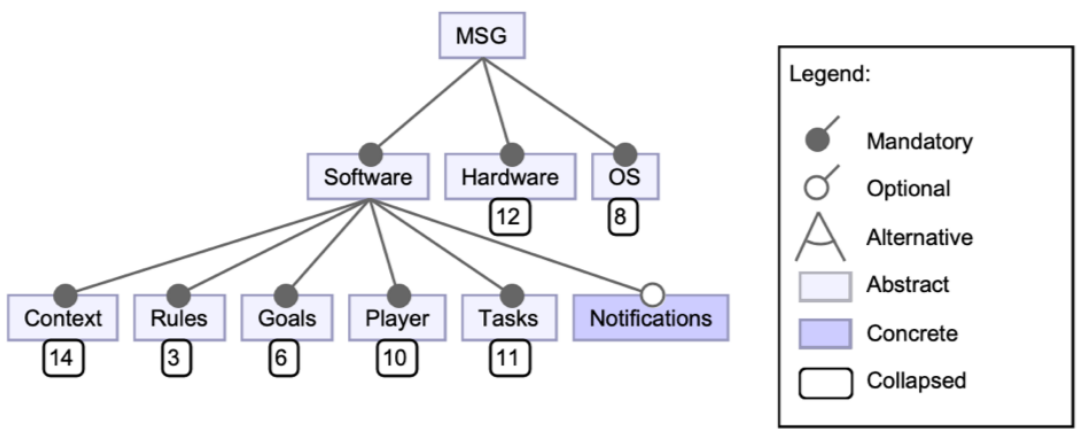

Fig. 3. The software part the of MSG Feature Model

Other differences also arise in the material part and operating system. All these variabilities must be clearly explained in order to facilitate the configuration of the serious game. For example, GPS systems are specific to certain mobile models or to the options selected when ordering. On the other hand, features such as Bluetooth may not exist in a given product, so they are modeled as optional features. The procedures related to the part of the operating system are generally different like Android and IOS, and especially strongly dependent on the version envisaged. Figure 4 shows the material part and MSG Feature Model operating system:

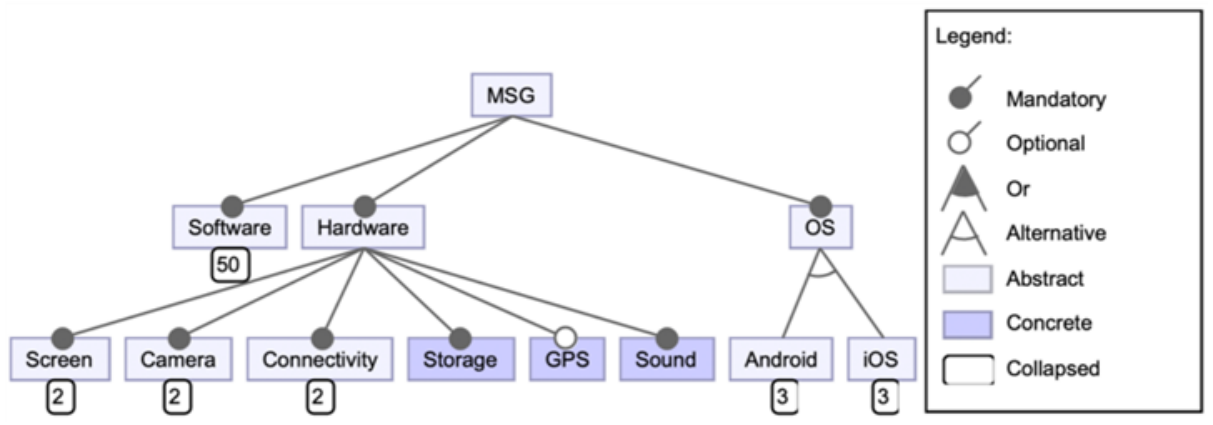

Fig. 4. The hardware and operating system part of the MSG Feature Model

These characteristics make it possible to distinguish products from each other. The variability analysis makes it possible to identify these features, to specify the constraints linking them and to explain the alternatives offered during the selection (reuse) of the features.

\section{Discussion}

After a bibliographical study, the following are the aspects that players considered to be most important for their experience of serious gambling: 


\subsection{Convenience of mobile devices}

An essential factor for serious game players is the hardware platform on which games are available. Today, most users are very comfortable with the use of mobile devices, even more so than with the more traditional desktop environment.

These remarks indicate that players place great importance on the comfort offered by mobile devices compared to other platforms. Players want the games to start quickly, so they can easily switch from one app to another and play games where and when they want.

\subsection{The player's attention}

The players indicated that it is sometimes difficult to concentrate on a serious game during a prolonged period and that they can be easily distracted by the notifications of their mobile devices or the other external events.

Besides the challenge to keep the attention of the player while playing the game, the developers of games have to make sure that the attention of the players is managed on the parts planned of the game; otherwise the players could miss important information.

It is important that the designers of games consider how they are going to keep the delighted players. It involves attracting the players so that they return to the game and, once inside the game, to keep them captivated and to center them on the concerned zones. For example, the uses of the notifications push to alert the players that new contents or surprises wait for them in the game.

\subsection{Feelings of the player to the contents of the subject}

The serious games postpone other video games in the sense that they contain of the material of learning which developers wish to pass on to the player. The players agreed that the context in which takes place the learning is very important. They considered that the learning is more effectively made in a relaxed environment where they can learn by playing.

The users also suggested that the world of the game could offer possibilities of accidental learning. The accidental learning makes reference to the acquisition of knowledge which are not explicitly taught, but not obtained by means of contextual indications. The researchers concluded from what precedes that the players want to learn effortlessly and without realizing really that they learn.

\subsection{Feelings of the players in front of challenges of the game}

The players regularly mentioned the need for motivation throughout the game. Numerous users called back to what extent the teachers had reprimanded them at the school while they did not know the teaching equipment or did not fight with them. It brought them to have negative associations with the material of learning, which dam- 
aged their learning. The participants considered that the games should avoid giving negative comments and rather encouraging, if a player struggles.

The participants insinuated that the positive reactions could take the shape of rewards and that these rewards motivated them to continue to play. The players will probably continue to play a game if they receive frequent rewards instead of penalties.

Certain participants also indicated that they wished more games - questionnaires in the game and the possibility to return on already ended games - questionnaires. To allow the players to return on the previous challenges allows them to practice and to improve their skills.

These observations suggest strongly that when the players are confronted with challenges in the game, the game should encourage them in a positive way if they struggle, to reward them if they manage the challenges and to give them the possibility of seeing them again to put into practice their skills.

\subsection{The feeling of the players to the world of the game}

The use of audio and visual elements in the game imposes the transfer of knowledge of the contents of the subject.

It emerges clearly from these remarks that the players like making continent of the game by using the largest number of their senses. The participants underlined colors, sounds, music and animation, which play all an essential role in the dumping in the game

Besides, the participants also indicated that they wished to know how they progressed in the world of the game, at the same time in terms of story of the game and the contents of learning. The players expressed the pleasure to know to what extent they progressed in the intrigue of the game and to what extent they again have to go before ending or winning the game.

The users also indicated that it was also essential to have relevant comments on the progress of the contents of the learning.

\subsection{Assistance to the serious games}

The participants considered that the game should supply an adequate assistance and they appreciated the supplied advice. In this context, the orientation concerns at the same time the navigation in the world of the game and the resolution of the riddles.

Some users argued that it might even be better to have facilitators available to provide advice during serious games, especially when children are the target audience.

The game should be designed to automatically determine the player's progress in order to provide relevant advice to the player. 


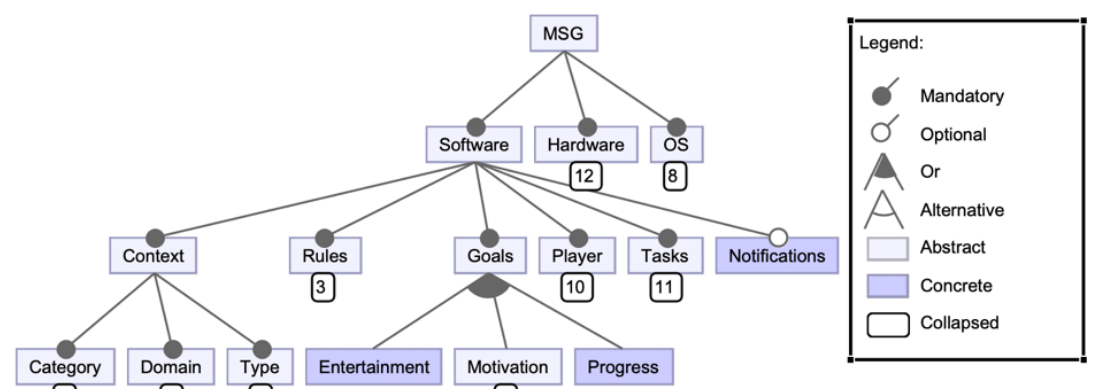

53

Fig. 5. Background details and game goals

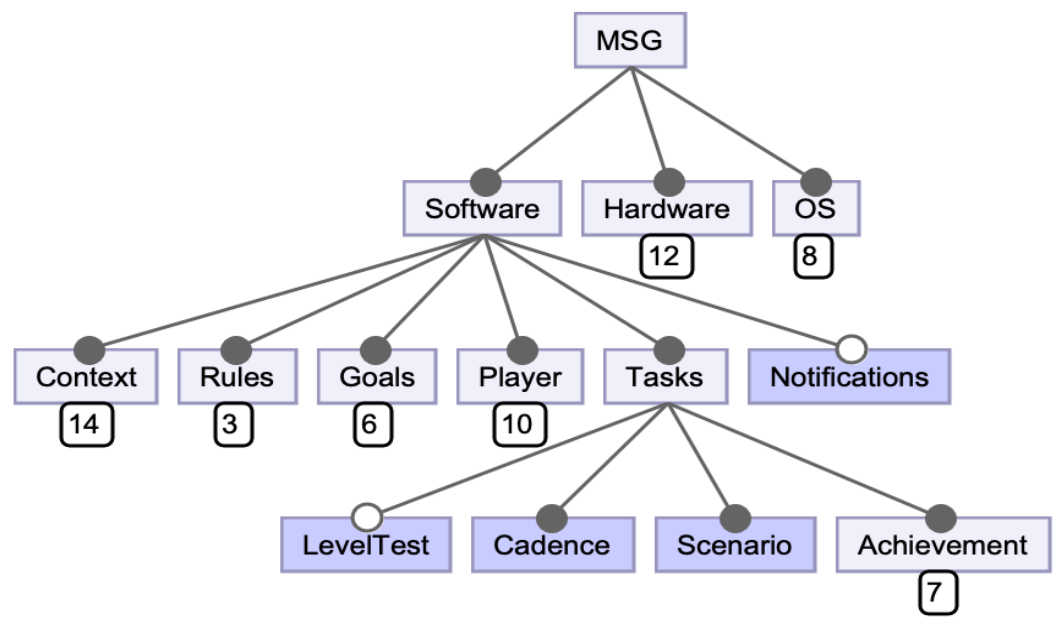

Fig. 6. Tasks and Game Rules

An important aspect of MSG is the challenge of adapting the game to the unique characteristics of each player. Players differed in gender, age, style of play and skill level and this greatly influences the gaming experience. Players with different skills will need different levels of guidance to make sure they find the game too easy or too hard.

Serious games are different from other video games in that their target market is generally very diverse and needs to adapt to different styles of players' game to succeed. The other games have a target audience generally more homogeneous. Incorporating elements to adapt different game styles based on existing taxonomies may allow players to better identify with the game and find it more interesting, as it corresponds to their own style of play.

Many aspects aimed at guiding developers in designing effective and fun MSG have been formulated. This research examined the experiences of players with serious games. These aspects can be further investigated in subsequent studies. 


\section{Conclusion}

In this article, the need to adopt the SPL approach is crucial to solving our problem. We have presented a modeling that can be easily applied to a set of MSGs belonging to different application domains. This work also focused on how players experience serious games. The aspects of serious gambling, that players considered the most important for their experiments, were identified.

The next work will be the implementation of the SPL domain for managing the variability of the different MSG contexts, all through asset construction (models, source code files, libraries, etc.) via tools such as Feature Oriented Modeling and Feature House.

\section{References}

[1] Apel, S., Batory, D., Kästner, C., \& Saake, G. (2016). Feature-oriented software product lines. Springer-Verlag Berlin An. https://doi.org/10.1007/978-3-642-37521-7 2

[2] Capilla, R., Bosch, J., Trinidad, P., Ruiz-Cortés, A., \& Hinchey, M. (2014). An overview of Dynamic Software Product Line architectures and techniques: Observations from research and industry. Journal of Systems and Software, 91, 3-23. https://doi.org/10.1016/ j.jss.2013.12.038

[3] Czarnecki, K., Grünbacher, P., Rabiser, R., Schmid, K., \& Wąsowski, A. (2012). Cool features and tough decisions: a comparison of variability modeling approaches. In Proceedings of the sixth international workshop on variability modeling of software-intensive systems (pp. 173-182). ACM. https://doi.org/10.1145/2110147.2110167

[4] Díaz, J., Pérez, J., \& Garbajosa, J. (2015). A model for tracing variability from features to product-line architectures: a case study in smart grids. Requirements Engineering, 20(3), 323-343. https://doi.org/10.1007/s00766-014-0203-1

[5] FeatureIDE, http://www.featureide.com/

[6] Lameras, P. (2015). Essential features of serious games design in higher education. Learning, 4(5).

[7] Rhouma, T. B. (2012). Composition of software product line models (Doctoral dissertation, Université Paris Sud-Paris XI).

[8] Thüm, T., Leich, T., \& Krieter, S. (2018). Feature Modeling and Development with FeatureIDE. Modellierung.

[9] Ziadi, T. (2004). Handling UML Product Lines (Doctoral dissertation, Rennes 1).

[10] Alvarez, J., Djaouti, D., \& Rampnoux, O. (2016). Learning with serious games?.

[11] Boudier, V. L., \& Dambach, Y. (2010). Serious game: Educational revolution.

[12] Djaouti, D., Alvarez, J., Jessel, J.-P. (2015). Classifying Serious Games: the G/P/S model.

[13] Barcenilla, J., \& Bastien, J. M. C. (2009). The acceptability of new technologies: what relationship with ergonomics, usability and user experience? Human labour, 72(4), 311-331.

[14] Brangier, E., \& Bastien, J. M. C. (2010). 12. The changing ergonomics of computer products: accessibility, usability, emotionality and influence. In Ergonomics, product design and mediatized services (pp. 307-328). University Press of France.

[15] Daumal, S. (2012). User Experience Design. UX Principles and Methods, 1-2.

[16] Kubicki, S., Borgiel, K., Lepreux, S., Wolff, M., \& Kolski, C. (2012). Reflections around interactive tables: user experience, usability, evaluation. Human work, 75(3), 229-252. https://doi.org/10.3917/th.753.0229 
[17] Moser, C. (2013). User experience design. In User experience design (pp. 1-22). Springer, Berlin, Heidelberg.

[18] Stevens, R., Polk, K., Merrill, C., Feng, F., Weiss, M., Brosnan, E., \& Barnes, L. E. (2018). User experience design to enhance the effectiveness of mobile technologies for the treatment of mental health. In Systems and Information Engineering Design Symposium (SIEDS), 2018 (pp. 135-140). IEEE. https://doi.org/10.1109/sieds.2018.8374724

[19] Fitchat, L., \& Jordaan, D. B. (2016). Ten heuristics to evaluate the user experience of serious games.

[20] Nacke, L. E., Drachen, A., \& Göbel, S. (2010). Methods for evaluating gameplay experience in a serious gaming context. International Journal of Computer Science in Sport, 9(2), $1-12$.

[21] Nagalingam, V., \& Ibrahim, R. (2015). User experience of educational games: A review of the elements. Procedia Computer Science, 72, 423-433. https://doi.org/10.1016/j.procs.20 15.12 .123

[22] Zielke, M. A., Zakhidov, D., Jacob, D., \& Lenox, S. (2016, July). Using Qualitative Data Analysis to Measure User Experience in a Serious Game for Premed Students. In International Conference on Virtual, Augmented and Mixed Reality (pp. 92-103). Springer, Cham. https://doi.org/10.1007/978-3-319-39907-2_9

[23] Zaharias, P., Gatzoulis, C., \& Chrysanthou, Y. (2012). Exploring user experience while playing educational games: Focus on temporality and attractiveness. International Journal of Gaming and Computer-Mediated Simulations (IJGCMS), 4(4), 19-32. https://doi.org/10. 4018/jgcms.2012100102

[24] Fitchat, L. (2016). Characterising HCI principles for evaluating the user experience of a serious game (Doctoral dissertation).

[25] Santana-Mancilla, P. C., Rodríguez-Ortiz, M., Gaytán-Lugo, L. S., \& García-Ruíz, M. Á. (2015). User Experience Evaluation to El Encanto: a Serious Game for Learning Community Intervention. Research in Computing Science, 108, 81-88.

[26] Le Marc, C., Mathieu, J. P., Pallot, M., \& Richir, S. (2010, June). Serious gaming: From learning experience towards User Experience. In 2010 IEEE International Technology Management Conference (ICE) (pp. 1-12). IEEE. https://doi.org/10.1109/ice.2010.747702 $\underline{8}$

[27] Moreno-Ger, P., Torrente, J., Hsieh, Y. G., \& Lester, W. T. (2012). Usability testing for serious games: Making informed design decisions with user data. Advances in HumanComputer Interaction, 2012, 4. https://doi.org/10.1155/2012/369637

[28] Radianti, J., Lazreg, M. B., \& Granmo, O. C. (2015). Fire simulation-based adaptation of SmartRescue App for serious game: Design, setup and user experience. Engineering Applications of Artificial Intelligence, 46, 312-325. https://doi.org/10.1016/j.engappai.2015. $\underline{06.012}$

\section{$7 \quad$ Authors}

Chaimae Meftah received an engineering diploma in Computer Science in 2014 from National School of Applied Sciences, Oujda, Morocco. She is a PhD student who works on Mobile Serious Games in MASI Laboratory, Mohammadia School of Engineers, Mohammed V University, Rabat, Morocco. Her main field of research interest is gamification based on Software Product Line and User eXperience. 
Asmaâ Retbi received an engineer degree in Computer Science in 1997, DESA degree in Computer Science in 2000, PhD in Computer Science With RIME team in 2015 at the Mohamed V University Agdal, Mohammadia School of Engineers (EMI); Professor at the Computer Science Department at the Mohammadia School of Engineers; Ongoing research interests: E-learning, Authoring tool, MDE and Domain Specific Modeling.

Samir Bennani received an engineer degree in Computer Science in 1982; Doctorate degree in Computer Science, $\mathrm{PhD}$ in Computer Science in 2005; Former chief of the Computer Science Department at the Mohammadia School of Engineers (EMI); Professor at the Computer Science Department-EMI; Ongoing research interests: SI, Modeling in Software Engineering, Information System, eLearning content engineering, tutoring, assessment and tracking.

Mohammed Khalidi Idrissi received a doctorate degree in Computer Science in 1986, PhD in Computer Science in 2009; Former Assistant chief of the Computer Science Department at the Mohammadia School of Engineers (EMI); Professor at the Computer Science Department-EMI; Ongoing research interests: SI, ontology, Web services, MDA, elearning and evalution

Article submitted 2019-05-20. Resubmitted 2019-07-30. Final acceptance 2019-08-07. Final version published as submitted by the authors. 